Biochem. Lett., Vol. 5, PP. 119-139 (2009)

\title{
RELATIONSHIP BETWEEN LEUKOCYTE COUNT, ERYTHROCYTE SEDIMENTATION RATE, AND TYPE 2 DIABETES MELLITUS
}

\author{
A. M. Afan, M. E. Zendah, H. M. Abu- Saida and \\ S. S. Alabdeli. \\ Faculty of Medicine, Al-Fateh University for Medical \\ Sciences.
}

\begin{abstract}
ABSRACT
Because few prospective studies have addressed this issue, this study examined the relation between DM type 2 and leukocyte count, ESR, BMI, BP. Total and differential WBC count, ESR. BMI and blood pressure for 250 participants: 91 men and 159 women. Among 91 men there are 62 diabetic, and among 159 female subjects, 110 develop diabetes were measured. All using data were collected from diabetic Hospital. In the present work a graded association between higher WBC and diabetes mellitus type 2 was observed after adjusting for age, sex. ESR, BMI, and BP were also significantly associated $(P<0.05)$ with DM type 2 . Smoking status has no real effect in the present data. These finding are consistent with the hypothesis that an activation of leukopoiesis may play a role in pathogenesis of DM type 2 and the inflammation is a dependent risk factor for diabetes. Additional studies are needed to determine whether circulating levels of inflammatory markers are associated with increased risk of incident diabetes.
\end{abstract}

\section{INTRODUCTION}

Diabetes is a progressive chronic disease that is emerging as a global epidemic. Diabetes imposes a tremendous burden on health economies mainly because of its devastating complications. Emerging 
data suggest that DM type 2 may play a role in the increasing level of leukocyte count and ESR. Data have been accumulated since as early as the 1960s that the systemic concentration of many immune mediators in the circulation appears chronically unregulated in diabetes. The link between type 2 diabetes and inflammation has been the research focus of many groups since 1997/1998 (2). It has been suggested that a chronic activation of immune system may play a role in the pathogenesis of diabetes. Whether leukocytes are involved in the pathogenesis of diabetes is still poorly known. However, there has been a little research on the relation between

WBC count and vascular complications of diabetes. In 1999, Schmidt and his colleagues showed that a variety of inflammatory markers, including white blood count are predict in the development of type 2 diabetes in middle-aged population. To date, there is some evidence from prospective studies support the hypothesis which stated that alteration markers of inflammation such as an increase in peripheral total WBC, neutrophil, monocyte, and lymphocyte count is associated with the development of type 2 diabetes and may be activated through the release of cytokines, such as TNF- alpha, transforming growth factor beta 1, monocyte chemoattractant protein 1 interlukins and others to participate in the pathogenesis of diabetic complications (12), (13), (1), (4). Surprisingly, Barbora et al, (1) showed that a high WBC was associated with a decline in insulin action.

Recently, many studies confirmed the direct association between the DM type 2 and leukopoiesis, for instance; a study of US adults, showed that leukocyte count is significantly associated with diabetes incidence (3), (8). Nicola Pannacciulli et al (2003) studied the relationship between WBC count and family history type 2 DM. They indicate that $\mathrm{WBC}$ count is increased in patient with genetic predisposition to type 2 DM. In 2004, Peter C.Tong et al, suggested that there is an association between WBC count and albuminuria in type 2 diabetes.

Few researches suggested that a chronic low-grade activation of the immune system particularly innate immune system is found to be closely involved in the pathogenesis of type 2 diabetes ${ }^{(3)}$. Moreover, Giovanni Solinas ${ }^{(5)}$ stated that impaired of an anti-inflammatory 


\section{Blood cells and type 2 diabetes mellitus}

response plays a role in the pathogenesis of type 2 diabetes. In addition, recent studies have shown that there is a relation between, erythrocyte sedimentation rate and diabetes mellitus type 2 as well.

It has been found that ESR is fairly strong associated with DM and increased by any cause of inflammation. Earl S. (3) stated that ESR can be predicting the risk of coronary heart disease and stroke. These alterations of the total WBC count and the elevation of the ESR could be predicting the future incidence of heart disease ${ }^{(7),(11),(9)}$.

Normally the basal ESR is highly higher in female than male. Although it appeared that the association between ESR and diabetes incidence was stronger among males and females, sex was not significant effect (William C.Shiel et al, 2007).

\section{MATERIAL \& METHODS}

Participants considered as a diabetic if: 1) they confirm that they had ever been told by a doctor that they have diabetes, 2) hospitalization record listed. Permission has been taken to obtain hospital records and to collect blood samples from diabetic patients. Blood was collected from diabetic patient as well as age matching nondiabetic subjects. Each subject participated in a detailed interview of his or her personal disease and smoking history. Information on health status of subjects was assessed by questionnaire. All subjects asked for their age, sex, cigarette smoking, body mass index (BMI) $\left(\mathrm{kg} / \mathrm{m}^{2}\right)$.

Peripheral leukocyte analyses included total leukocyte count and differential percentages of neutrophils, monocytes, lymphocytes, eosinophils, and basophils (lymphocyte \& neutrophil were considered only) using automated cell counter (Sysmex KX 21 CBC machine). To minimize the confounding effect of infection, subjects with a WBC count $<4 \times 10^{3} / \mathrm{cmm}$ or $>10 \times 10^{3} / \mathrm{cmm}$ were rechecked for the analyses. Any specimen with abnormal or atypical leukocytes was reanalyzed and excluded. The laboratory analyses were under internal control of the hospital. 


\section{Determination of ESR:}

To perform the manual ESR test in the study, a Westergren method was used. This method was performed by using a Westergren calibrated tube (or pipette) which is graduated from $0-150 \mathrm{~mm}$ and a Westergren rack with ten pipettes for holding the tubes. An anticoagulant substance (EDTA) was added to a sample of $5 \mathrm{ml}$ blood and placed in the Westergren tube of standard dimensions. All tubes were incubated in a vertical position for exactly one hour. At the end of the hour, the distance of erythrocytes have fallen from the plasma at zero mark is measured in millimeters $(\mathrm{mm})$ and reported. The distance that the red cells sediment down through the plasma in 1 hour is taken to be the ESR, the data will be reported in $\mathrm{mm} / \mathrm{hr}$.

\section{Statistical Analysis:}

The analysis was performed using the SPSS (version 10.1) statistical package. All data are expressed as $\pm \mathrm{SE}$ or median as appropriate. Student's $t$ test was used for comparisons between-group for continuous variables. $\mathrm{P}<0.05$ was considered to be significant.

\section{RESULTS}

The present data showed that in male and female, DM type 2 has significant effect on the total WBC count and the percentages of differential WBC (lymphocyte only), BMI, and ESR, however, no significant interactions were present between DM and BP in both sexes except in female's SBP it shows significant effect compared with the normal group $(\mathrm{p}<0.05)$.

Figure $1 \& 2$ shows that WBC count tended to be higher in diabetic males than females compared with the normal. The results show also that diabetic patients (especially female) had significant elevation of lymphocytes concentration compared with the normal one $(P<$ $0.0001, P<0.05$ ) (Fig $3 \& 4$ ). However, no significant effect what so ever was found on the number of circulating neutrophils in both sexes (Fig 4\&5). Date shows also that diabetic patients had a significant higher ESR than participants who remained free of diabetes. It 
appeared that there is a very strong association between ESR and DM type 2 among males $(P<0.0001)$ (Fig 7$)$ than females $(P<0.05)$ (Fig $8)$.

From the obtained results it seems to be that there is a direct relationship between Dm type 2 and body weight. From calculation of the BMI, figure $9 \&$ figure 10 showed that male and female diabetic patient had a significantly higher BMI than normal $(P<0.05)$, this is not true for male BP (figure 11), however, in female, figure 12 shows significant elevation in systolic BP but not diastolic BP $(P<0.05)$.

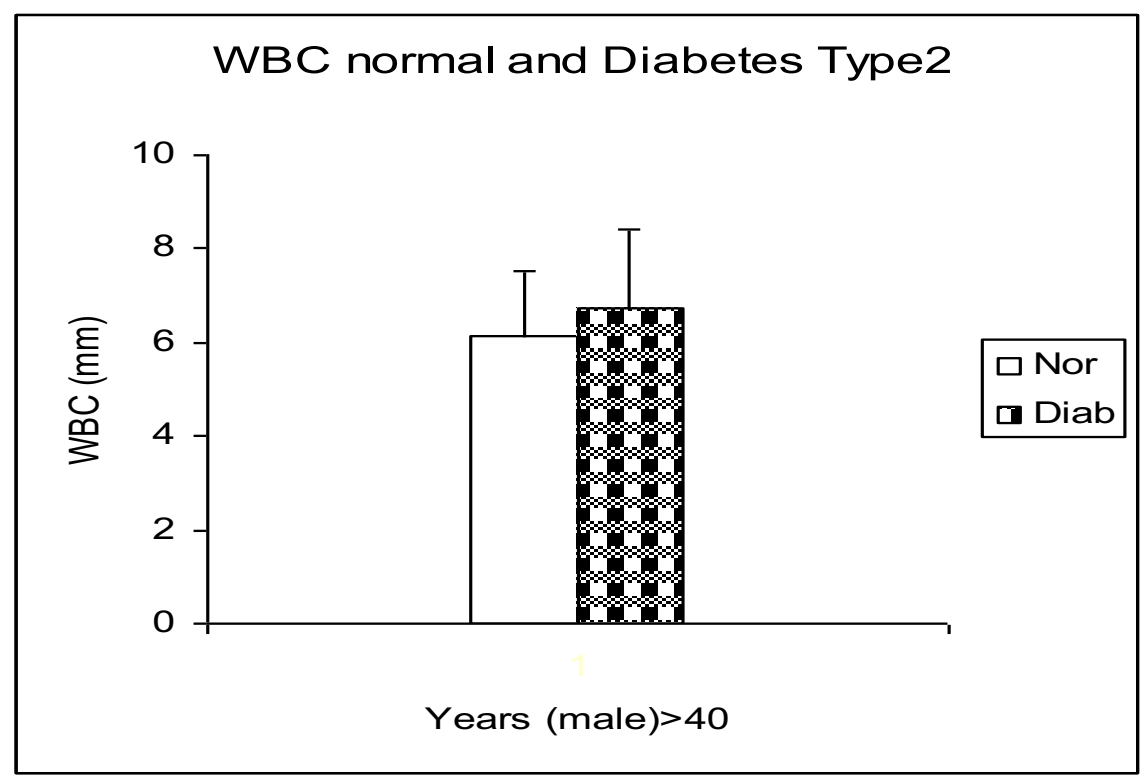

123

Fig.1: Values are expressed as mean \pm SD of subjects (male), shows total WBC in two different groups, Normal (hollow bars) and Diabetic (square bars). Values between groups are significantly different at: $*=$ $(P<0.05)$. 


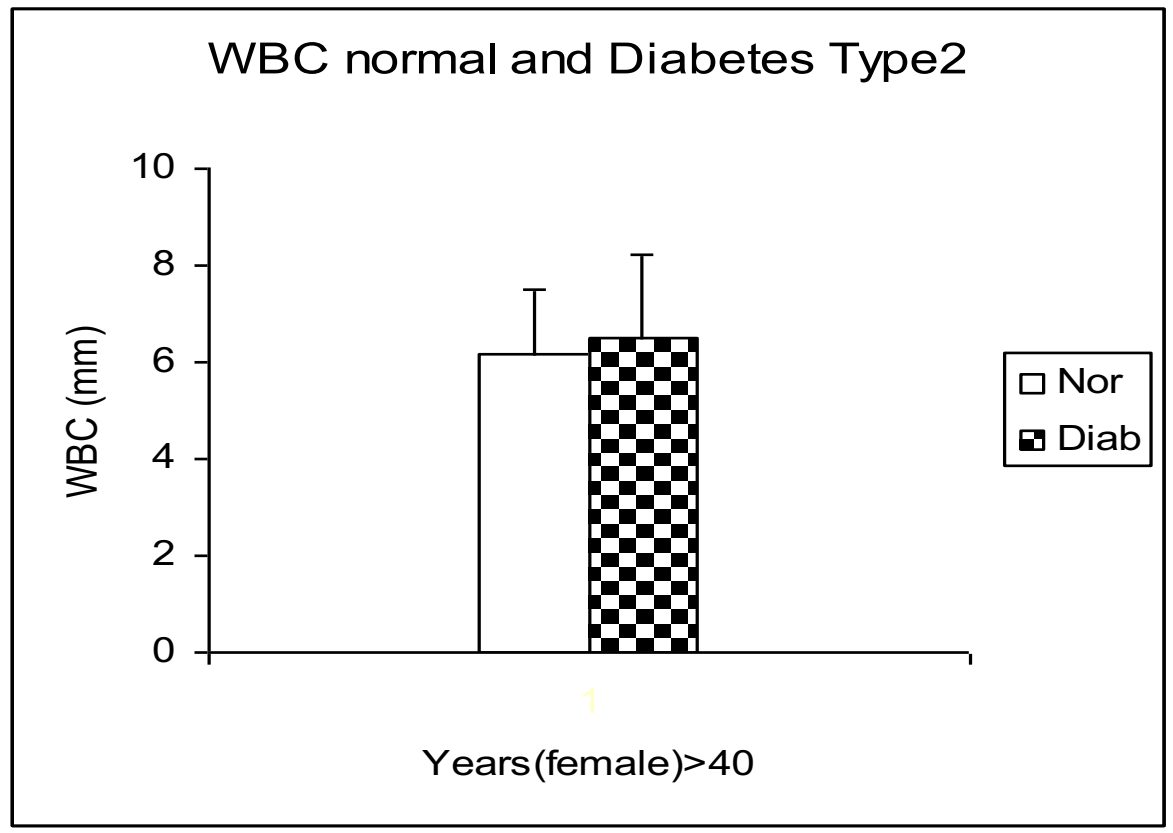

Fig.2: Values are expressed as mean $\pm \mathrm{SD}$ of subjects (female), shows total WBC in two different groups, Normal (hollow bars) and Diabetic (square bars). Values between groups are significantly different at: $\quad *=(\mathrm{P}<0.05)$. 
Blood cells and type 2 diabetes mellitus

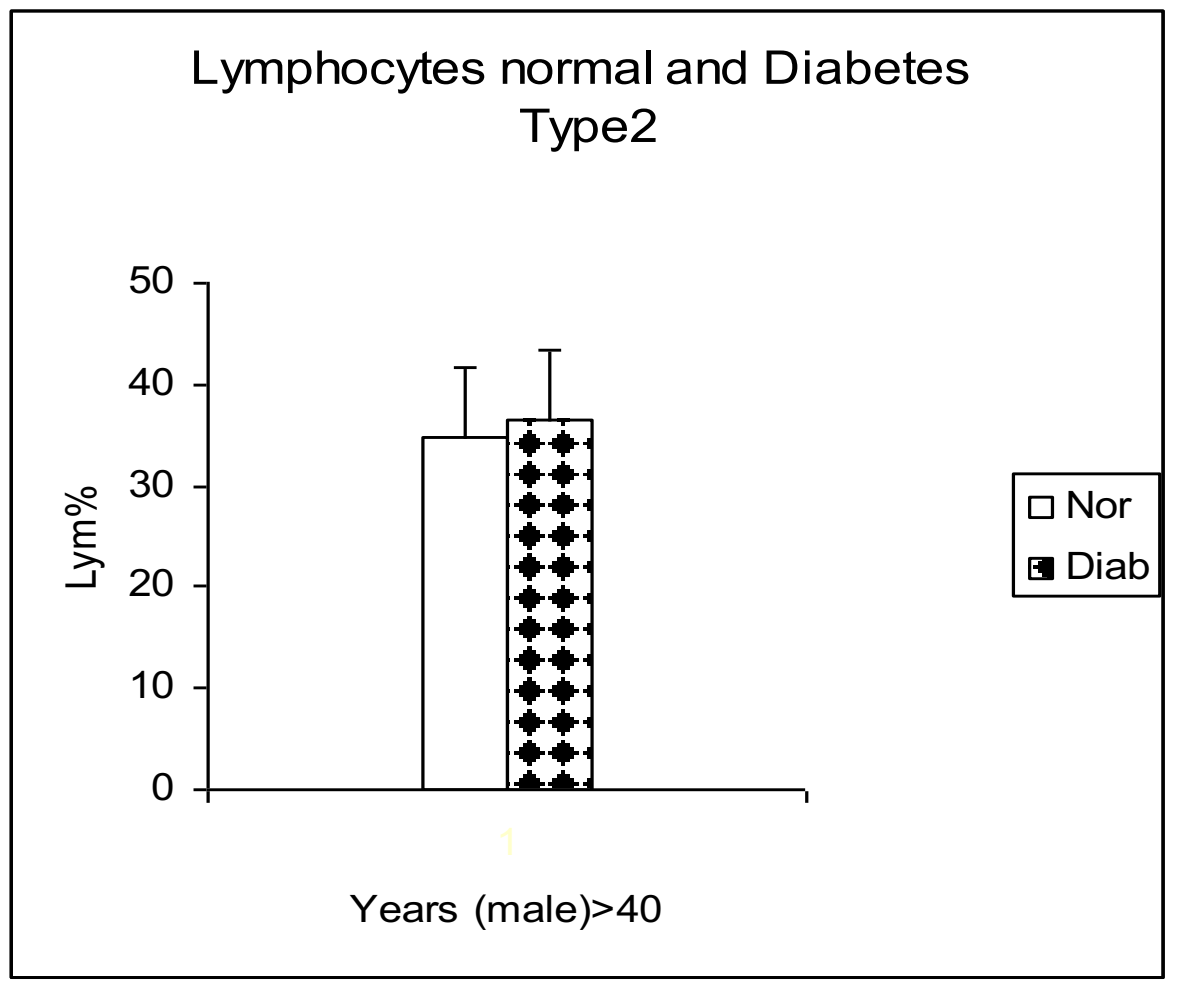

Fig.3: Values are expressed as mean \pm SD of subjects (male); shows lymphocytes percentage in two different groups, Normal (hollow bars) and Diabetic (filled bars). Values between groups are significantly different at: ${ }^{*}=(P<0.05)$. 


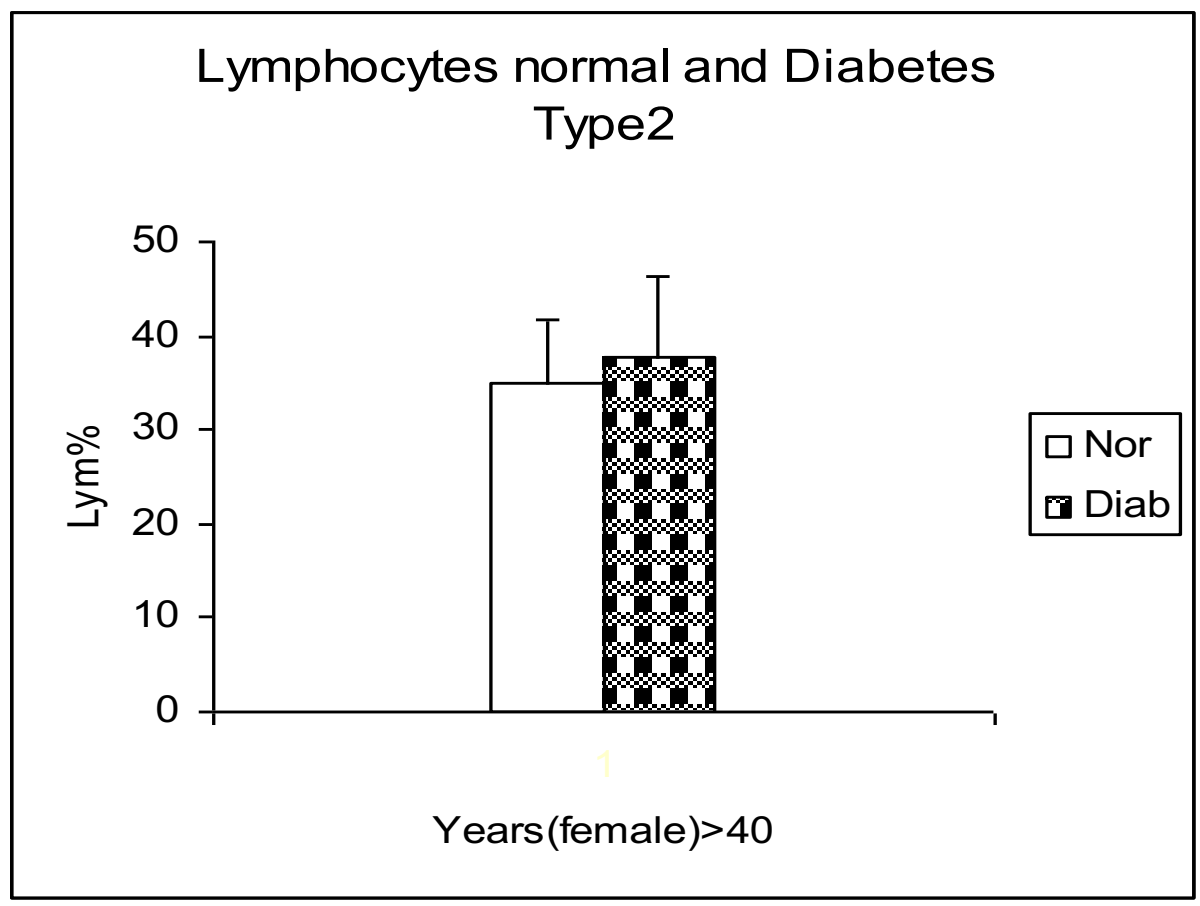

Fig.4: Values are expressed as mean \pm SD of subjects (female); shows lymphocytes percentage in two different groups, Normal (hollow bars) and Diabetic (square bars. Values between groups are significantly different at: $*=(P<0.05)$. 


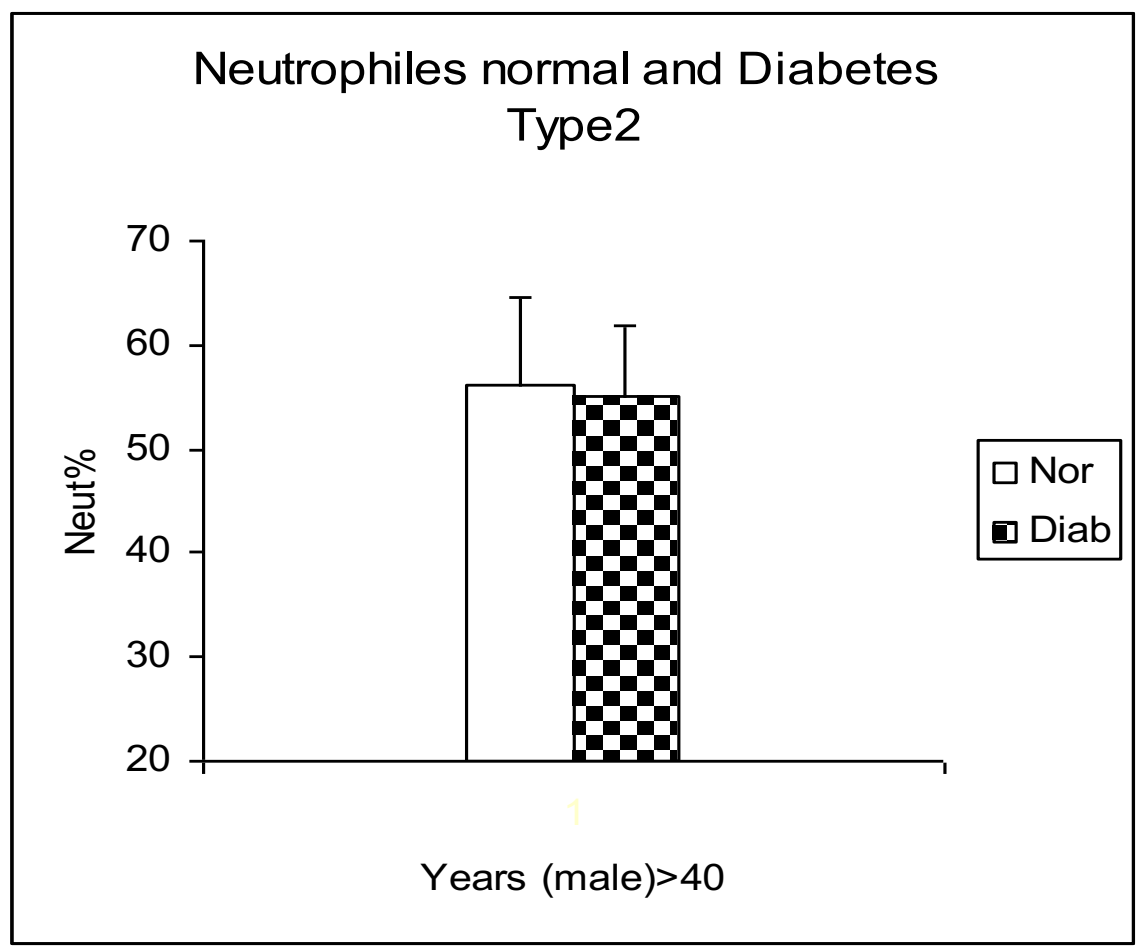

Fig.5: Values are expressed as mean \pm SD of subjects (male); shows neutrophiles percentage in two different groups, Normal (hollow bars) and Diabetic (square bars). Values between groups are significantly different at: $*=(P<0.05)$. 
Afan, A. M. et al.

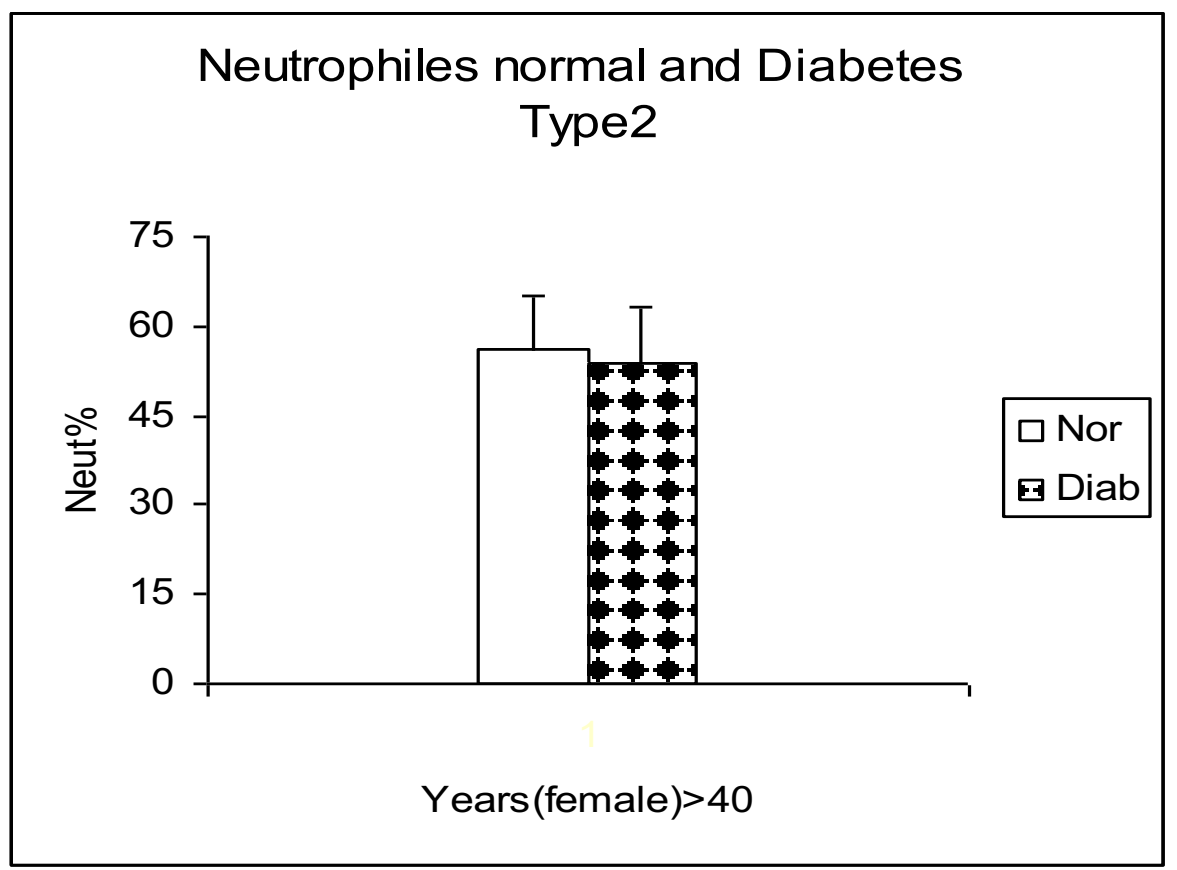

Fig.6: Values are expressed as mean $\pm \mathrm{SD}$ of subjects (female), shows neutrophiles percentage in two different groups, Normal (hollow bars) and Diabetic (filled bars). Values between groups are significantly different at: $*=(P<0.05)$. 
Blood cells and type 2 diabetes mellitus

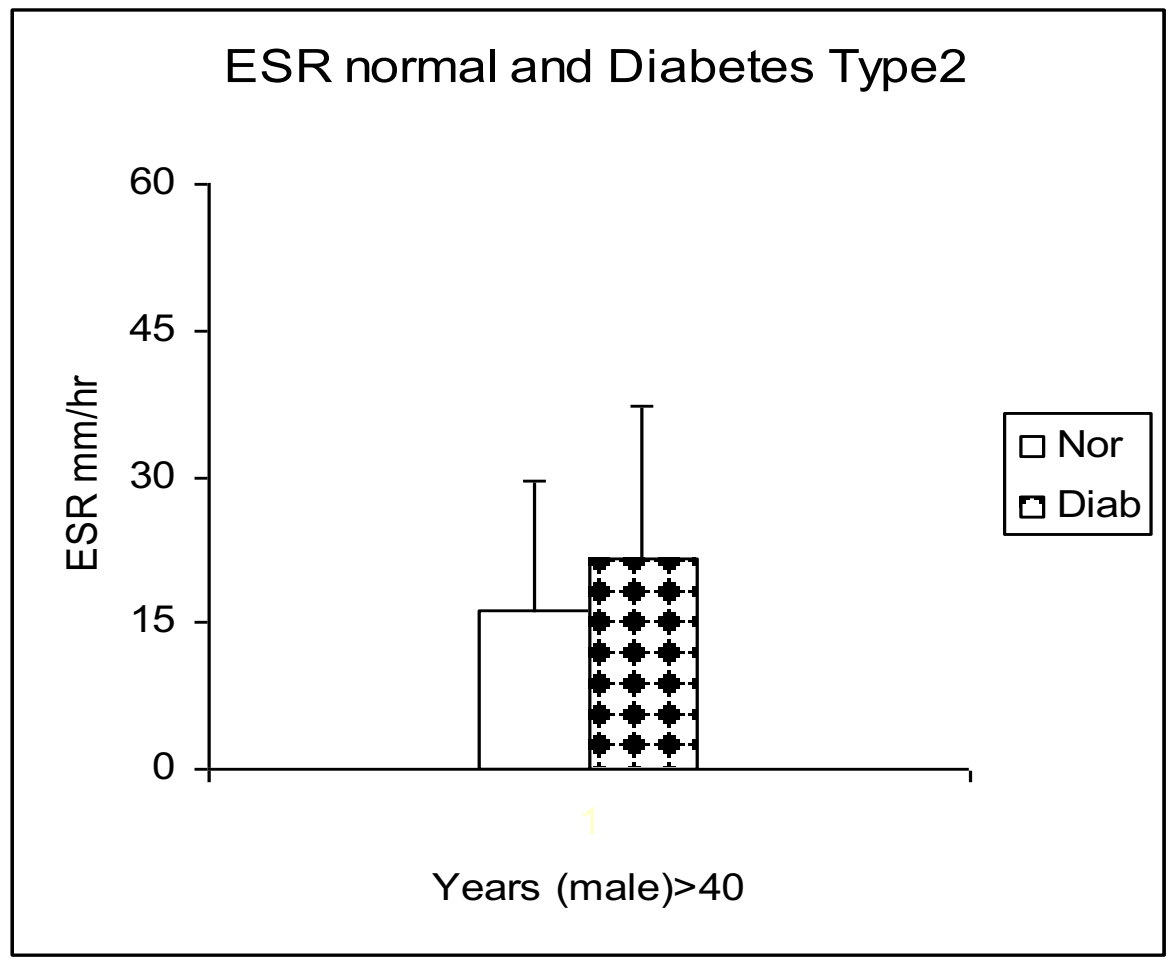

Fig.7: Values are expressed as mean \pm SD of subjects (male); shows ESR in two different groups, Normal (hollow bars) and Diabetic (filled bars). Values between groups are significantly different at: $\quad *=(P<0.05)$. 
Afan, A. M. et al.

\section{ESR normal and Diabetes Type2}

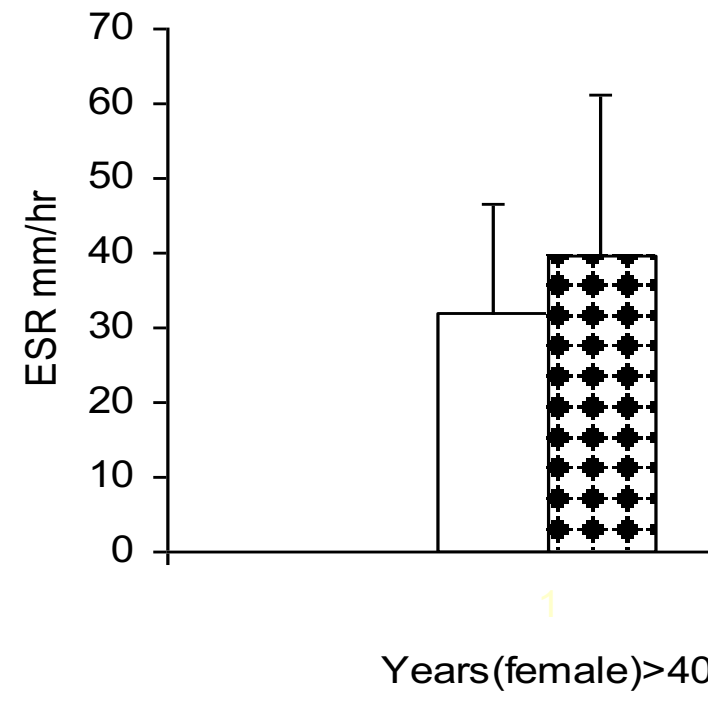

Fig.8: Values are expressed as mean $\pm \mathrm{SD}$ of subjects (female); shows ESR in two different groups, Normal (hollow bars) and Diabetic (filled bars). Values between groups are significantly different at: $*=(P<0.05)$. 
Blood cells and type 2 diabetes mellitus

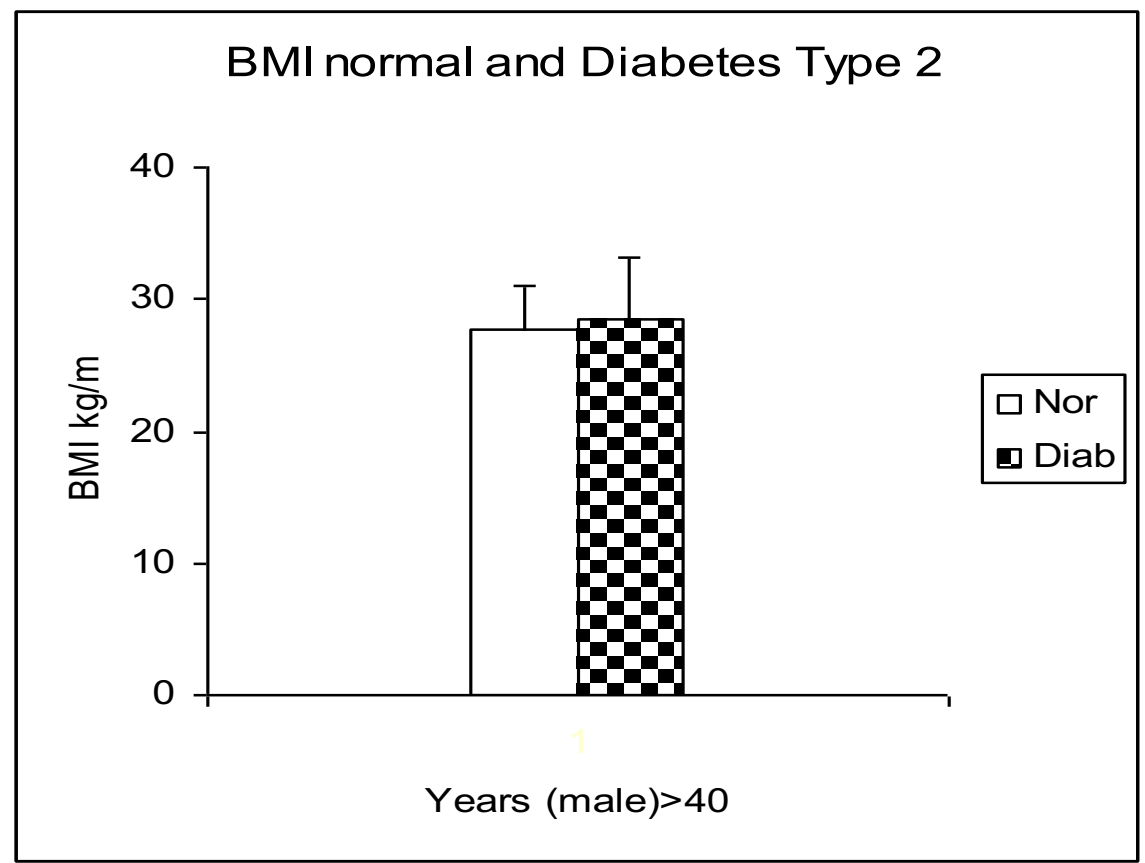

Fig. 9: Values are expressed as mean \pm SD of subjects (male); shows BMI in two different groups, Normal (hollow bars) and Diabetic (square bars). Values between groups are significantly different at: $*=(P<0.05)$. 
Afan, A. M. et al.

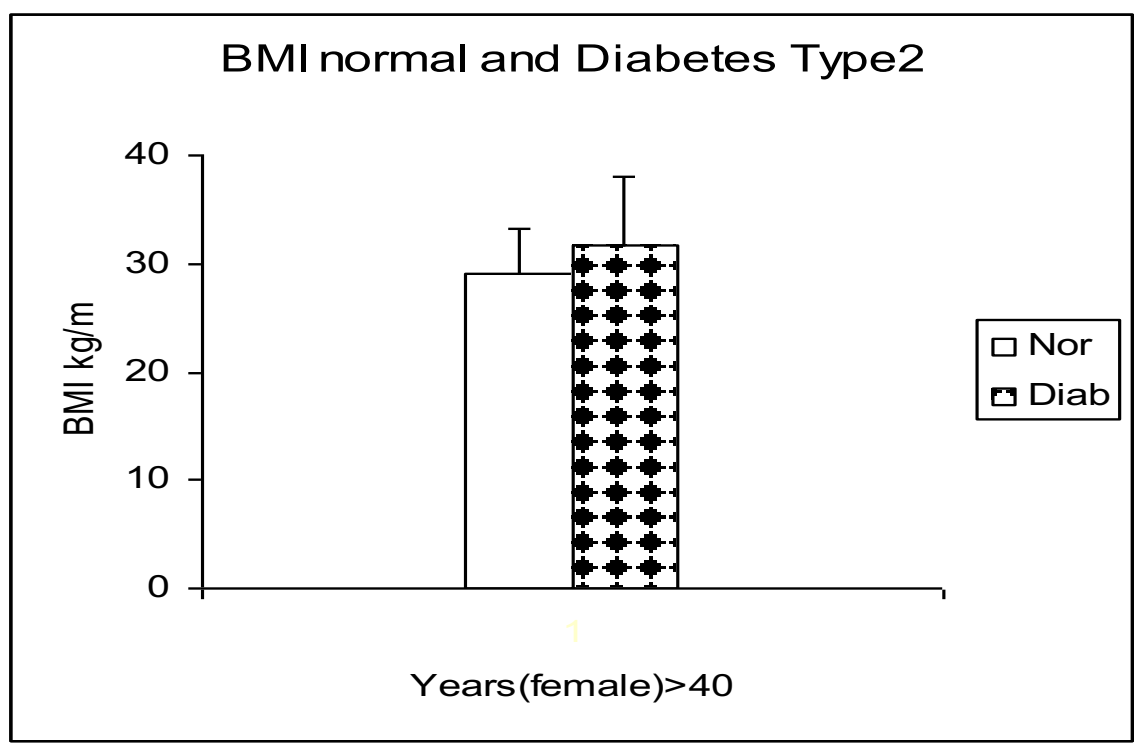

Fig.10: Values are expressed as mean \pm SD of subjects (female); shows BMI in two different groups, Normal (hollow bars) and Diabetic (filled bars). Values between groups are significantly different at: $*=(P<0.05)$. 


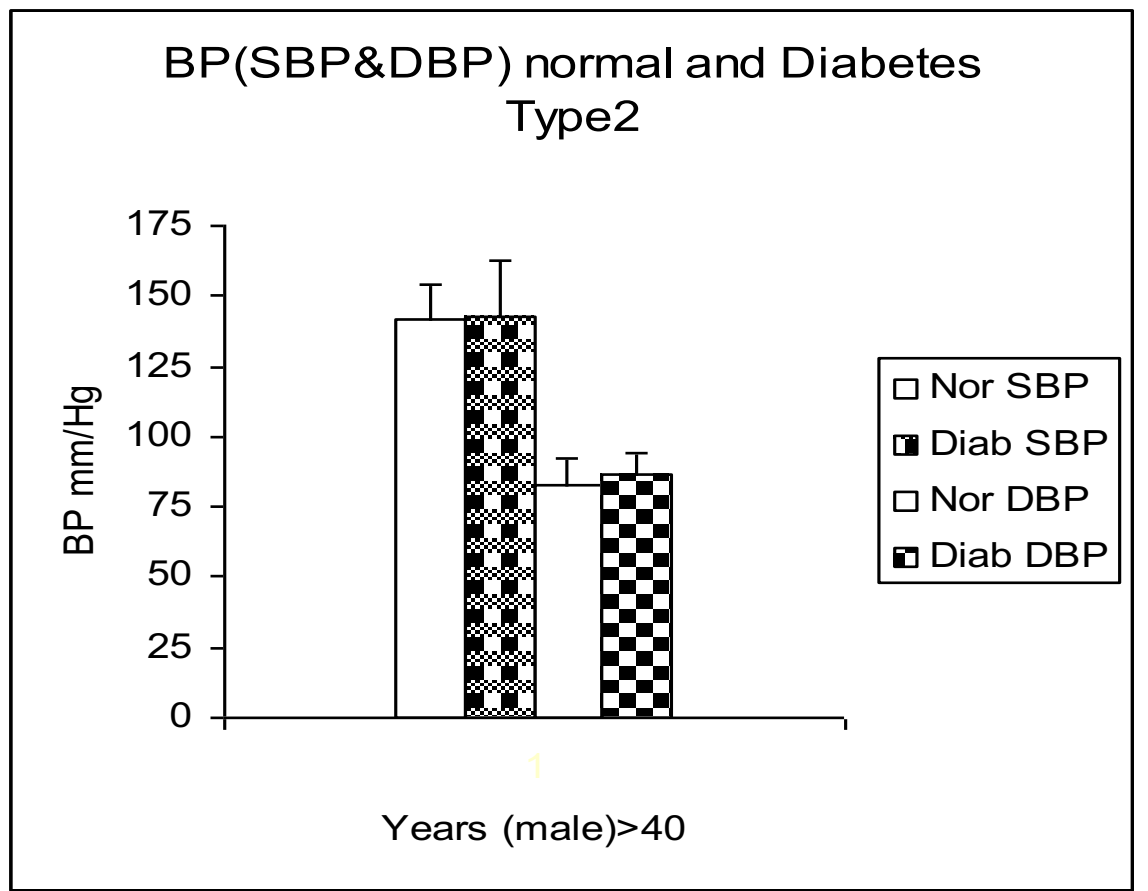

Fig.11: Values are expressed as mean \pm SD of subjects (male), shows BP (SBP\&DBP) in two different groups, Normal (hollow bars) and Diabetic (square bars). Values between groups are significantly different at: $*=(P<0.05)$. 


\section{BP(SBP\&DBP) normal and Diabetes Type2}

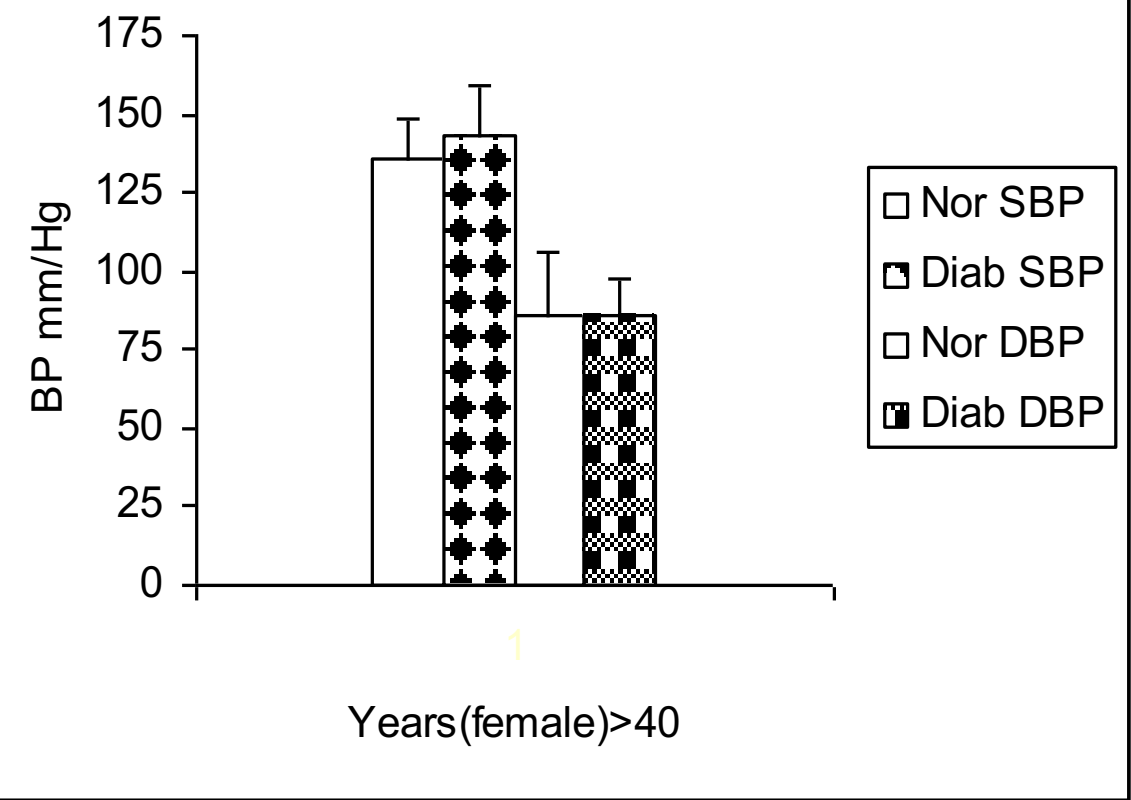

Fig.12: Values are expressed as mean \pm SD of subjects (female); shows BP (SBP\&DBP) in two different groups, Normal (hollow bars) and Diabetic (filled and square bars). Values between groups are significantly different at: $*=(P<0.05)$. 
Blood cells and type 2 diabetes mellitus

Table I : Shows that in male and female, DM type 2 has significant effect on the total $\mathrm{WBC}$ count and the percentages of differential WBC (lymphocyte only), BMI, and ESR, however, no significant interactions were present between DM and BP in both sexes except in female's SBP it shows significant effect compared with the normal group $(p<0.05)$.

1: Normal males

\begin{tabular}{|c|c|c|c|c|c|c|c|c|}
\hline & Age & Lym $\%$ & Neut $\%$ & WBC & ESR & BMI & SBP & DBP \\
\hline Mean & 55.42 & 34.90 & 56.15 & 6.14 & 16.13 & 27.64 & 141.80 & 83.20 \\
\hline S.D & 11.97 & 6.81 & 8.36 & 1.39 & 13.56 & 3.34 & 12.06 & 9.00 \\
\hline S.E & 0.93 & 0.53 & 0.64 & 0.1 & 1.23 & 0.32 & 1.16 & 0.54 \\
\hline
\end{tabular}

2: Normal females

\begin{tabular}{|c|c|c|c|c|c|c|c|c|}
\hline & Age & Lym $\%$ & Neut $\%$ & WBC & ESR & BMI & SBP & DBP \\
\hline Mean & 50.90 & 35.03 & 55.98 & 6.16 & 31.74 & 29.00 & 135.47 & 85.57 \\
\hline S.D & 11.94 & 6.68 & 9.07 & 1.36 & 14.93 & 4.21 & 13.14 & 20.56 \\
\hline S.E & 0.8 & 0.45 & 0.54 & 8.5 & 0.99 & 0.27 & 0.98 & 0.56 \\
\hline
\end{tabular}

3: Diabetic males type 2

\begin{tabular}{|c|c|c|c|c|c|c|c|c|}
\hline & Age & Lym $\%$ & Neut\% & WBC & ESR & BMI & SBP & DBP \\
\hline Mean & 56.44 & 36.47 & 55.03 & 6.75 & 21.67 & 28.49 & 142.57 & 86.38 \\
\hline S.D & 10.41 & 6.98 & 6.73 & 1.66 & 15.56 & 4.74 & 20.27 & 7.66 \\
\hline S.E & 0.73 & 0.4 & 0.48 & 7.48 & 0.88 & 0.24 & 0.91 & 0.49 \\
\hline
\end{tabular}

4: Diabetic females type 2

\begin{tabular}{|c|c|c|c|c|c|c|c|c|}
\hline & Age & Lym $\%$ & Neut $\%$ & WBC & ESR & BMI & SBP & DBP \\
\hline Mean & 56.83 & 37.78 & 53.97 & 6.48 & 39.79 & 31.62 & 142.74 & 85.74 \\
\hline S.D & 10.59 & 8.45 & 9.26 & 1.73 & 21.34 & 6.47 & 16.01 & 11.89 \\
\hline
\end{tabular}


Afan, A. M. et al.

\begin{tabular}{|l|l|l|l|l|l|l|l|l|} 
S.E & 0.65 & 0.36 & 0.44 & 0.25 & 0.84 & 0.23 & 0.81 & 0.46 \\
\hline
\end{tabular}

\section{DISCUSSION}

Thus, leukocyte count and ESR, which are perhaps specific indicators of inflammation, could be used to provide limited support to emerging data that suggest that inflammation may impact the pathogenesis of diabetes. Further research is required to establish the causal relation of $\mathrm{WBC}$ and diabetic complications and the underlying mechanisms. It would be interesting to measure the serial changes of differential WBC counts to further clarify the role of WBC's in the development of diabetes complications. It would also be useful to know if the WBC measurements are stable over time. The bias effect of such measurements should be attenuated by a larger number of patients enrolled.

Mounting evidence implicates inflammation in the pathogenesis of type 2 diabetes. It was not possible to determine the cause of increased WBC from data collected in the current study. One possible explanation is that both a higher $\mathrm{WBC}$ and diabetes reflect an underlying activation of the immune system. In the current study, we observed a positive and graded association between total and differential WBC count and DM type 2. Although there are only few researches reporting the differential leukocyte count in relation to diabetes, this study clarifies that the composite members of $\mathrm{WBC}$, especially the lymphocyte, is associated with DM 2 suggesting that WBC count, may play a linkage role in the development of DM 2. The data show also that leukocyte count and ESR are significantly and positively related to diabetes mellitus. According to this we suggest that type $2 \mathrm{DM}$ can exert influences on the bone marrow to produce large number of WBC and circulate them. No statistical significant associations were found between DM and neutrophil count. These results are generally in agreement with previous reports ${ }^{(1,6)}$.

On the other hand, Earl S. (3) mentioned that ESR was not significantly associated with DM after adjustment of the data for various possible confounders. But at the same time ESR showed 


\section{Blood cells and type 2 diabetes mellitus}

strong association with diabetes incidence among only men. In this study, however, ESR showed direct related with DM among men and women. In addition, the present data showed that there is a direct relationship between DM and the body weight.

The same finding was reported by Earl S. ${ }^{(3)}$ who stated that an increase in WBC's count predicted weight gain. We believe that diabetic patient who have high BMI, they show high BP, but unexpected results was notified. No significant change in diabetic BP was seen compared with the normal except in female SBP. Moreover, smoking status in this research did not appear to have an additive effect on WBC count.

The mechanisms through which an increased WBC count might increase a person's risk of DM remain to be elucidated. One possibility is explained this relation which is; the activation of the immune system causes the decline in insulin sensitivity and, therefore, contributes to the development of DM. More specifically, perhaps leukocytes particularly lymphocytes, might contribute directly to harming the beta cells of the pancreas that are responsible for the production and secretion of insulin.

In addition, the raised WBC count may reflect the effects of a lowgrade inflammation initiated by obesity or other mechanisms (3). Moreover, people with diabetes are at increased risk of developing infections that can also raise concentration of proinflammatory cytokines and fibrinogen therefore ESR will be raised. Surprisingly Peter C. ${ }^{(10)}$ showed that WBC count, even within the normal range, was independently associated with complications of diabetes. This finding is not agreement with current studies. Fu-Mei Chung et al, ${ }^{(4)}$ suggested other mechanisms contributing to leukocytosis in diabetic patients. These mechanisms are related to plasma cortisol and changing insulin level.

Both factors are known to increase WBC counts by increasing neutrophil influx from marrow storage. Most likely the same finding was obtained by this work which showed increase in lymphocyte count instead of neutrophil. These findings are consisting with the hypothesis that chronic activation of the immune system may play a 
role in the pathogenesis of DM. We thought that prospective analysis revealed that a high total and differential WBC predicts type 2 diabetes, and this in agreement with findings obtained by many populations ${ }^{(1)},(4)$.

In overall, the obtained date in this research comes to that; an elevation of total WBC count and, ESR value in patients with DM type 2 is support the theory that inflammation has a role in the etiology of DM. We were unable to examine associations with DM specifically, an outcome that could be more relevant to the pathophysiology of DM. Hence, several potential thinking to the current study deserves consideration. Additional studies are needed to confirm these results and determine the risk of DM type 2 associated with inflammation. Such findings could help to identify novel risk factors for DM risk and potential targets for prevention and treatment.

\section{REFERENCES}

1- Barbora Vazarova, Christian Weyer, Robert S. Lindsay, Richard E. Pratley, Clifton Bogardus, and P. Antonio Tataranni. (2002): High White Blood Cell Count Is Associated with a worsening of Insulin Sensitivity and Predicts the Development of Type 2 Diabetes. Clinical Diabetes and nutrition Section, National Institutes of Health, 4212 N. 16 th street. Rm. 541, phoenix, AZ 85016.

2- C. Herder, T. Illig, W. Rathmann, S. Martin, B. Hastert, S. Müller-Sholze, R. Holle, B.Thorand, W. Koenig, H.E. Wichman, H. Kolio. (2005): Inflammation and Type 2 Diabetes: Results from KORA Augsburg. Vol 67. No 1: S115-S121.

3- Earl S. Ford. (2002): Leukocyte count, Erythrocyte Sedimentation Rate, and Diabetes Incidence in a National Sample of US Adults. American Journal of Epidemiology. Vol 155. No 1.pp 57-64.

4- Fu- Mel Chung, MS , Jack C.R, T sai, MD, Dao- Ming Chang ,MD, Shyi-Jang Shing, MD. PHD, Yau-Jiunn Lee, MD, PHD. (2005): Periripheral Total and Differential Leukocyte Count in Diabetic Nephropathy. Diabetes Care. Vol 28, No 7: 1710-1717. 
5- Giovanni Solinas., (2007): Type 2 Diabetes: Inflammation, Not Obesity, Cause of Insulin Resistance. University of California San Diego. (UCSD) School of medicine.

6- Laveryl A, Armstrong D. C. Quabedea U xtl. Walker S. C. (1996): Puncture wounds: normal laboratory values in the face of severe infection in diabetics and non-diabetics. The American Journal of medicine. Vol. 101. No 5, pp 521-525.

7- Madjid M. Awan I, Willerson JT, Casscells SW., (2004): Leukocyte count and coronary heart disease: implications for risk assessment. J Am cardiol. No 44: 1945-1956.

8- Nicola Pannacciulli, Francesco Giorgino, Raffaele A. Martina. (2003): White blood cell count and family History of diabetes Diabetes. Nutrition Research Newsletter. Vol 11. No.10: 12321237.

9- Olivares R, Ducimetiere P, Clande JR. (1993): Monocyte count: a risk factor for coronary heart disease? Amj Epidemiol. No. 137:49-53.

10- Peter C., Tong, PHD, Ka-Fai Lee, MBCHB, Wing-Yee So, MBCHB, Margaret H. Ng, K. Lo, MBCHB, Norman N. Chan, MD and Juliana C. Chan, MD. (2004): White Blood Cell Count Is Associated With Macro-and Micro Vascular Complications in Chines Patients with Type 2 Diabetes. Diabetes care. No. 27: 216222.

11- Prentice RL, Szatrowski TP, Fujikura T, Kato H , Mason Mw, Hamilton H H . (1982): Leukocyte count and coronary heart disease in Japanes cohort. Am j Epidemiol. No 116: 496-509.

12- Scherberich JE. (2003): Proinflammatory blob monocytes: main effector and target cells in systemic and renal disease; background and therapeutic implications (Review).

Intjclin pharmacol Ther. No 41: 459-464.

13- Shanmngam N, Redy MA, Guhan M, Natarajan R. (2003): High glucose induced expression of proinflammatory cytokine and chemokine genes in monocytic cells. Diabetes No. 52: 1256-1264.

14- William C. Shiel, Jr, MD, Facp, Facr. (2007): Erythrocyte Sedimentation rate. American Family physician article on ESR. 